Illinois State University

ISU ReD: Research and eData

Theses and Dissertations

4-22-2015

\title{
Switching tactics: Phenotypic plasticity in the alternative mate- finding tactics of burying beetles
}

Theresa Mulrey

Illinois State University, pieninta@gmail.com

Follow this and additional works at: https://ir.library.illinoisstate.edu/etd

Part of the Biology Commons, and the Ecology and Evolutionary Biology Commons

\section{Recommended Citation}

Mulrey, Theresa, "Switching tactics: Phenotypic plasticity in the alternative mate-finding tactics of burying beetles" (2015). Theses and Dissertations. 402.

https://ir.library.illinoisstate.edu/etd/402

This Thesis is brought to you for free and open access by ISU ReD: Research and eData. It has been accepted for inclusion in Theses and Dissertations by an authorized administrator of ISU ReD: Research and eData. For more information, please contact ISUReD@ilstu.edu. 


\title{
SWITCHING TACTICS: PHENOTYPIC PLASTICITY IN THE ALTERNATIVE MATE-FINDING TACTICS OF BURYING BEETLES
}

\author{
Tess E. Piening Mulrey
}

33 Pages

August 2015

Male Nicrophorus burying beetles utilize two alternative mate-finding tactics, representing an ideal model system to test the influence of environmental cues on the expression of alternative tactics. The "searching" tactic involves flying in search of a carcass on which to mate. This tactic can result in high levels of paternity, but is risky in that carcasses are rare and competition for carcasses is fierce. The "signalling" tactic, which involves emitting pheromone in the absence of a carcass to attract females, is energetically less costly, but a signalling male must mate with multiple females to achieve the same reproductive returns as a successful searcher. Most male burying beetles employ both tactics, but the amount of time spent employing each tactic differs between individuals. Because all males are phenotypically plastic in the time invested in alternative behaviours, the expression of the tactics is likely to be condition dependent. While size has been shown to have an effect on tactic expression, it does not explain all of the variation. As a conditional-strategy, an environmental cue, such as the availability of reproductive resources, may influence the "switchpoint" of when one tactic yields higher fitness than the other.

We tested how the expression of alternative tactics is influenced by previous mating experience and the perceived availability of females versus carcasses by using a repeated- 
measures design in which mate-finding behaviour of males was observed before and after exposure to: 1) multiple females but no carcasses, 2) multiple females and multiple carcasses, 3) multiple carcasses but no females, or 4) no females or carcasses (control). We found a significant time-by-treatment interaction for time invested in signalling, with both the control and multiplefemale groups significantly increasing signalling time. Although the overall time-by-treatment interaction was not quite significant for time invested in searching, pairwise comparisons revealed that control, multiple-female, and multiple-carcass groups all significantly decreased time spent searching. Our results demonstrate that environmental cues do affect which alternative tactic male burying beetles employ, and that the chosen tactic is influenced by the availability of both receptive females and carcasses on which to breed. 
SWITCHING TACTICS: PHENOTYPIC PLASTICITY IN THE

\title{
ALTERNATIVE MATE-FINDING TACTICS
}

OF BURYING BEETLES

TESS E. PIENING MULREY

\author{
A Thesis Submitted in Partial \\ Fulfillment of the Requirements \\ for the Degree of \\ MASTER OF SCIENCE \\ School of Biological Sciences \\ ILLINOIS STATE UNIVERSITY
}

2015 
C 2015 Tess E. Piening Mulrey 
SWITCHING TACTICS: PHENOTYPIC PLASTICITY IN THE ALTERNATIVE MATE-FINDING TACTICS

OF BURYING BEETLES

TESS E. PIENING MULREY

COMMITTEE MEMBERS:

Scott K. Sakaluk, Co-chair

Anne-Katrin Eggert, Co-chair

Steven A. Juliano

Ben M. Sadd 


\section{ACKNOWLEDGMENTS}

I would first and foremost like to thank Scott K. Sakaluk and Anne-Katrin Eggert for all of their help and guidance with this project and for aiding my development as a scientist. I am especially thankful to Anne for teaching me the ways of the burying beetle and for giving her time to personally help with data collection. I could not have asked for better mentors. I would also like to thank committee members Ben M. Sadd and Steven A. Juliano for their input and support with this project.

Many others helped me along the way: Thanks to Jim Dunham for donating his time and skills to help build observation chambers, to Sarah Kluk for helping with beetle care, to E. Keith Bowers and Amanda Wilson Carter for statistics advice, and to Kristin Duffield for moral support and laughs the whole time. I owe many thanks as well to my husband Patrick, not only for supporting me in every way possible, but also for volunteering his time to help with this project.

Finally, I thank all of my family and friends, near and far, for encouragement, laughter, and motivation. I dedicate this work to my nephew Leo Brown; I hope to inspire in him wonder and awe of all things wild, even the creepy-crawlies.

T. P. M. 


\section{CONTENTS}

$\begin{array}{lr} & \text { Page } \\ \text { ACKNOWLEDGMENTS } & \text { i } \\ \text { CONTENTS } & \text { ii } \\ \text { TABLES } & \text { iii } \\ \text { FIGURES } & \text { iv } \\ \text { CHAPTER } & \end{array}$

I. SWITCHING TACTICS: PHENOTYPIC PLASTICITY IN THE ALTERNATIVE MATE-FINDING TACTICS OF BURYING BEETLES 1

$\begin{array}{ll}\text { Abstract } & 1\end{array}$

Introduction $\quad 3$

$\begin{array}{ll}\text { Methods } & 9\end{array}$

Experimental Animals $\quad 9$

Experimental Design $\quad 11$

Statistical Analyses $\quad 15$

Results 15

Discussion $\quad 17$

$\begin{array}{ll}\text { Funding } & 26\end{array}$

$\begin{array}{ll}\text { REFERENCES } & 27\end{array}$ 


\section{TABLES}

Table $\quad$ Page

1. Repeated Measures Mixed-Model ANCOVA F-tests for Effects of Treatment,Time, Treatment*Time, and Pronotum Length of Signalling and Searching.

2. Signalling and Searching Were Repeatable, Both Before and After the Treatment Period. 


\section{FIGURES}

Figure

Page

1.Total Number of Observations Spent Signaling (Solid Line) and Searching (Dashed Line) Pre- and Post-Treatment for the Four Treatment Groups. The * Indicate a Significant Difference Between Pre-Treatment and Post-Treatment Values. 


\title{
CHAPTER I
}

\section{SWITCHING TACTICS: PHENOTYPIC PLASTICITY IN THE ALTERNATIVE MATE-FINDING TACTICS OF BURYING BEETLES}

\begin{abstract}
Male Nicrophorus burying beetles utilize two alternative mate-finding tactics: searching and signalling. The searching tactic involves flying in search of a carcass on which to mate, while the signalling tactic involves emitting pheromone in the absence of a carcass to attract females. All males employ both tactics, but the amount of time spent employing each tactic differs between individuals. Because all males are phenotypically plastic in the time spent employing alternative behaviours, the expression is likely to be condition dependent on some environmental cue, such as the availability of reproductive resources (i.e. females and carcasses). We tested how the expression of alternative tactics is influenced by previous mating experience and the perceived availability of females versus carcasses by using a repeated-measures design in which mate-finding behaviour of males was observed before and after exposure to: 1) multiple females but no carcasses, 2) multiple females and multiple carcasses, 3) multiple carcasses but no females, or 4) no females or carcasses (control). We found a significant time-by-treatment interaction for time invested in signalling, with both the control and multiple-female groups significantly increasing signalling time. Although the overall
\end{abstract}


time-by-treatment interaction was not quite significant for time invested in searching, pairwise comparisons revealed that control, multiple-female, and multiple-carcass groups

all significantly decreased time spent searching. Our results demonstrate that environmental cues do affect which alternative tactic male burying beetles employ, and that the chosen tactic is influenced by the availability of both receptive females and carcasses on which to breed.

Keywords: alternative tactics, phenotypic plasticity, conditional strategy, Nicrophorus, behavioural strategy, experience 
Introduction

Alternative mating behaviours, prevalent across a wide range of taxa, are characterized by multiple distinct behavioural phenotypes of the sexually selected sex, typically males (Waltz \& Wolf, 1984; Shuster, 2010). While alternative behaviours are often the result of intense sexual selection, they can evolve whenever a subset of males are excluded from mating even if sexual selection is not particularly intense (Shuster \& Wade, 2003).

There is ambiguity in the literature with respect to the definitions of key terms pertaining to alternative phenotypes. The term "strategy" is often used to describe both alternative phenotypes and the rules governing their expression, a double usage that can lead to confusion. Here, we will use the terminology advocated by Dominey (1984), in which a "strategy" is an evolved set of rules governing the expression of behavioural phenotypes, or "tactics". Thus, each individual has a single strategy, but there may be more than one tactic within this strategy (Dominey, 1984). To be considered an evolutionarily stable strategy, the strategy must achieve higher fitness than any alternative when expressed by a critical proportion of the population.

Alternative phenotypes can be maintained in the population as either mixed, alternative, or conditional strategies (reviewed in Gross, 1996; Dominey, 1984). Mixed strategies are those in which the alternative tactics are probabilistically assigned, with either every individual employing the alternative tactics in the same ratio or with individuals being permanently assigned tactics with set probabilities $p$ and 1-p (Maynard 
Smith, 1982; Dominey, 1984, Gross, 1996). When a genetic polymorphism results in the co-occurrence of multiple distinct behavioural morphs within a population at a stable mixture, such strategies are considered "alternative strategies". In this case, each morph is an allelic variant adopting a single-tactic strategy, and these morphs are expressed at specific proportions such that the average fitnesses of the morphs are equal in an evolutionarily stable state (Parker, 1982; Dominey, 1984; Gross, 1996; Shuster \& Wade, 2003), as observed in marine isopods, Paracerceis sculpta (Shuster \& Wade, 1991; Shuster \& Sassman, 1997) and side-blotched lizards, Uta stansburiana (Sinervo \& Lively, 1996).

In the case of both alternative and mixed strategies, each individual only expresses one tactic or expresses multiple tactics in a pre-determined ratio. However, most alternative reproductive behaviours studied to date consist of multiple tactics expressed in a condition-dependent manner (Dominey, 1984). These "conditional strategies" are characterized by alternative phenotypes that are influenced by an environmental cue or some aspect of the male's condition or status (Gross, 1996; Lee, 2004; Shuster \& Wade, 2003; Tomkins \& Hazel, 2007). Conditional strategies occur when there is a certain threshold or switchpoint at which males switch from expressing one tactic to the other; this switchpoint could be an intrinsic quality of the individual such as size or an extrinsic environmental cue (Shuster \& Wade, 2003; Tomkins \& Hazel, 2007). 
While some conditional strategies are characterized by an irreversible developmental switch, as documented in salmonid fishes (Aubin-Horth \& Dodson, 2004; Piché, Hutchings, \& Blanchard, 2008), many others exist in which shifts between tactics are rapid and reversible. This specific type of conditional strategy is termed a "behavioural strategy" and is characterized by phenotypically plastic males that can switch between tactics based on an extrinsic cue such as their relative status in the population (as in the status-dependent selection model in Gross, 1996) or another environmental cue such as population density or resource availability (Brockmann, 2001; Shuster \& Wade, 2003).

Such phenotypically plastic behavioural strategies are most likely to evolve when males have few mating opportunities, but when the availability of mating opportunities can be predicted by the environment (Shuster \& Wade, 2003). In this case, males will perceive the environment as "fine-grained", as it may change rapidly and multiple times throughout an individual's life (Levins, 1968; Brockmann, 2001; Shuster \& Wade, 2003). In the context of male mating behaviours, the environmental grain may be determined by the spatiotemporal distribution of females (Shuster \& Wade, 2003). Environments in which the circumstances surrounding a successful reproductive attempt are highly variable may also result in phenotypically plastic mating behaviours (Shuster \& Wade, 2003).

Alternative tactics comprising behavioural strategies are known throughout diverse taxa. Commonly, the alternative phenotypes are expressed in response to the 
immediate environmental conditions, as when individuals adjust their tactic according to their status relative to competitors. However, instances may exist in which the current environment is not indicative of the reproductive landscape as a whole and provides little information on which tactic use is optimal. Such may be the case with alternative matefinding tactics, where all males find themselves in a similar immediate environment devoid of reproductive resources at the time they have to decide which tactic to employ. In this case, males may use information gained from past experiences with respect to the availability of reproductive resources in making their decision on which tactic to employ.

Male Nicrophorus burying beetles represent an ideal model with which to test whether individuals use experience in decisions about tactic use. To successfully reproduce, burying beetles require a small vertebrate carcass, a rare and ephemeral resource. The rarity and value of this resource is indicated by the fact that individuals of both sexes will engage in sometimes deadly agonistic encounters, and that losers of fights often remain nearby the carcass, assuming "satellite" or brood parasitic status rather than locating a new carcass (Müller, Eggert, \& Dressel, 1990). That satellite males and brood parasitic females stay despite their lower reproductive success on the carcass is further indicative of the scarcity of this resource (Müller, Braunisch, Hwang, \& Eggert, 2007).

The two alternative mate-finding tactics employed by male burying beetles are to actively fly in search of a carcass on which to meet a female and raise a brood, or to emit pheromone in the absence of a carcass (henceforth termed "signalling"; Müller \& Eggert, 1987). If a searching male is able to successfully locate a carcass, he may be presented 
with a number of different situations, depending on the presence of other beetles. If another male is already present, the two fight over possession of the resource (Müller et al., 1990; Sakaluk \& Müller, 2008). If a female is present on the carcass, the pair bury and prepare the carcass, mating repeatedly in the process. When no females are present on the carcass when the male arrives, he superficially buries the carcass and emits pheromone to aid in attracting a female (Pukowski, 1933; Eggert, 1992; Wilson \& Fudge, 1984), which might inadvertently attract conspecific males or congeners of either sex who may attempt to usurp the resource (Müller \& Eggert, 1987; Eggert \& Sakaluk, 2000; Haberer, Schmitt, Shreier, \& Müller, 2011). While this tactic is risky, it can have high payoff; in N. vespilloides, the average paternity on a single carcass for a successful searching male is likely to be $84 \%$, with paternity up to $92 \%$ if there are no satellite males present (Eggert, 1992).

The signalling tactic is unlikely to yield as great of returns per brood, but likely has lower costs in terms of risky agonistic encounters and energetic demands. Females are attracted to the scent of the pheromone emitted by signalling males, and they cannot determine whether a male holds a carcass until they come into physical contact (Eggert \& Müller, 1989a; Eggert \& Müller, 1989b). Upon meeting a signalling male, the female allows a single copulation before flying away (Eggert \& Müller, 1989b), likely to ensure an adequate supply of viable sperm in case she locates a carcass on which another male is not present (Eggert, 1992). Field studies have found that between 19 and 39\% of females raise broods without a mate, presumably using sperm stored from mating with signalling males (Eggert, 1992; Scott \& Traniello, 1990; Müller et al., 2007). It is estimated that 
signalling males can achieve 5\% paternity of a single clutch if they are the last male to mate with a female (Eggert, 1992). However, their actual success depends on how long it takes for a female to subsequently locate a carcass, how many additional males she mates with, and whether another male is present on the carcass. It follows that a signalling male must mate with many females to achieve similar reproductive returns as a searcher. In spite of the difference between single-clutch paternities between the two tactics, a field study of $N$. vespilloides estimated that up to $45 \%$ of all larvae were sired by signalling males (Eggert, 1992; Müller et al., 2007).

All male burying beetles employ both tactics, but the proportion of time spent employing each tactic differs between individuals. While there is genetic variation underlying these behaviours, it does not account for all of the variation, and there is even substantial variation in behaviour within an individual (Eggert, 1992). Thus, these behavioural phenotypes are best viewed as alternative tactics within a conditional strategy. As a conditional strategy, there should be intrinsic or extrinsic cues that influence tactic expression. Previous studies have found that small males signal more frequently than large males, and that the influence of this intrinsic cue is not contingent on interactions with other beetles (Beeler et al., 1999; Walling et al, 2009). However, it is likely that there are also extrinsic environmental cues that influence a male's relative investment in the alternative tactics.

Because the reproductive success of male burying beetles is dependent on the spatiotemporal distribution of females and carcasses, experience with these resources 
may provide a valuable cue, allowing males to determine which tactic is likely to have greater returns in their specific environment. We tested this hypothesis by observing the behaviour of naïve male $N$. orbicollis before and after exposure to different reproductive experiences. We predicted that if males are able to gain information about the distribution of resources from their experience, they should adjust the relative amount of time invested in each tactic accordingly. Specifically, males that are allowed to mate with multiple females in the absence of a carcass may perceive a greater availability of potential mates; we predicted that these males would increase investment in signalling and decrease investment in searching. Males that encounter multiple carcasses should perceive a greater abundance of carcasses, which may yield higher returns for searching. We predicted that males encountering carcasses would increase investment in searching and decrease investment in signalling. However, if a male encounters a carcass in nature but is unable to attract a female, the carcass is of no reproductive value. Thus, we exposed males to carcasses with females either present or absent, to determine whether the presence of a female affects a male's perception of reproductive opportunities and differential investment in the alternative tactics.

\section{Methods}

\section{Experimental Animals}

Nicrophorus orbicollis occurs throughout eastern North America, primarily inhabiting hardwood forests. Experimental individuals were second- and third- generation 
lab-reared descendants of beetles caught in baited pit-fall traps in autumn 2013 at the Parklands Nature Preserve near Lexington, Illinois (40.6657N, 88.897W). Field-caught individuals were brought into the lab and maintained in plastic deli dishes with moist paper towels as a substrate, either individually or with another individual of the same sex. The beetles were fed small pieces of ground beef twice per week, at which time the paper towels were replaced with clean ones.

Field-caught beetles were paired randomly for breeding. Each pair was placed in a breeding chamber with a defrosted previously frozen 20 -g mouse carcass. When at least one member of the pair began inspecting the carcass, the beetles and the carcass were transferred to another container with moist peat. The pairs were then placed in a darkroom and allowed to mate, bury and prepare the carcass, and raise larvae. Once larvae dispersed from the carcass, they were removed from the breeding container. Up to five sibling larvae were placed into containers filled with about $4 \mathrm{~cm}$ of packed clean moist peat to pupate. These containers were kept in a dark incubator at $16^{\circ} \mathrm{C}$ until adults eclosed. The newly emerged beetles were sexed and placed in a new container with about $2 \mathrm{~cm}$ of moist peat, either individually or with one same-sex sibling, and maintained in an incubator on a $16 \mathrm{~L}: 8 \mathrm{D}$ photoperiod at $21^{\circ} \mathrm{C}$. They were fed two decapitated mealworms twice weekly, and the peat in their containers was replaced every other week.

Upon maturity, $F_{1}$ adults were randomly mated with unrelated individuals of the opposite sex to create an $\mathrm{F}_{2}$ population as described above. Larvae and newly emerged adults were treated as described above for the $\mathrm{F}_{1}$ generation, except that all larvae were 
housed individually until eclosion. As adults, male beetles were housed individually to avoid any variation in social environment, whereas females were housed with one or two same-sex siblings. These same procedures were also used to create an $F_{3}$ population. All $\mathrm{F}_{2}$ and $\mathrm{F}_{3}$ adults were maintained in incubators on a $16 \mathrm{~L}: 8 \mathrm{D}$ photoperiod at $21^{\circ} \mathrm{C}$, with males and females in separate incubators. These adult beetles were maintained in the same manner as described for the $\mathrm{F}_{1}$ adults.

\section{Experimental Design}

All beetles used in this study were sexually mature $F_{2}$ and $F_{3}$ adults, ranging in age from 29 to 59 days post-eclosion on the first day their behaviour was observed. The experiment was done in three rounds of 60 males, as this was determined to be the largest manageable number of beetles to observe at one time. Sexually mature male beetles $(N=180)$ were first weighed to the nearest milligram on a Mettler PE360 Delta Range digital scale balance, then placed individually into observation chambers. Observation chambers were lidded rectangular acrylic glass boxes $(20 \mathrm{~cm} \times 10 \mathrm{~cm} \times 10 \mathrm{~cm})$, with a small hole drilled about $1 \mathrm{~cm}$ from the top on each end to allow for gas exchange and to facilitate feeding. Observation chambers were filled with about $2 \mathrm{~cm}$ moist peat substrate and kept in a darkroom on the same 16L:8D photoperiod to which the beetles were acclimated. Red LED lights remained on in the darkroom 24 hours. After being placed in the observation chambers, each beetle was fed half a mealworm and allowed to acclimate to the chamber and the darkroom for 15-18 hours before observations began. 
Observations started 2 hours after 'lights off' and continued for 6 hours, until 'lights on' because pilot studies had shown that beetles were most active during this time. Beetle behaviour was scan-sampled at 10-minute intervals during this time. Males were recorded as either signalling, searching, or inactive during each scan. Males were scored as signalling if they were in the characteristic "head-stand" posture, with their abdomen extended upwards and their final abdominal segment fully extruded. Males were considered to be searching if they were flying, digging, walking, or running. If a male was stationary, but not signalling, he was scored as inactive.

After an observation period ended, each beetle was fed half a mealworm, which was inserted through one of the holes drilled in the side of the chamber to minimize disturbances associated with removing the lid. Observations were carried out on four consecutive days to establish each male's baseline signalling and searching frequencies. At the end of day four, each male was randomly assigned to one of four treatment groups: 1) control; 2) multiple-female; 3) multiple-carcass; and 4) female-and-carcass. An equal number of siblings from each family were assigned to each treatment to avoid potential confounding effects of genetic variation on signalling and searching frequencies (see Eggert, 1992).

After the pre-treatment observations were complete, males were transferred individually to small deli containers containing about $2 \mathrm{~cm}$ of moist peat substrate. Males in the multiple-carcass and female-and-carcass treatment were provided with a thawed 20-g mouse carcass; a single female was also added to the containers with males in the 
female-and-carcass treatment. All containers were transferred to incubators set to the same 16L:8D photoperiod for the duration of the seven-day treatment period. The control and multiple-female groups were kept in a separate incubator from the carcass treatments to prevent these males from receiving any olfactory cues from the carcasses.

Males in the two treatments with carcasses were allowed to remain on a carcass for three days, and the males in the female-and-carcass group were allowed to mate with the female and bury and prepare the carcass with her. After these three days, all males in these treatments were transferred into new deli containers with about $2 \mathrm{~cm}$ of moist peat, and given one day to recover before repeating the process again. Thus, in the seven-day treatment period, males in the multiple-carcass treatment were given two carcasses in succession and males in the female-and-carcass group were given two carcasses and two females in succession.

In the multiple-female treatment, males were given access to three females for 45 hours per day. These females were added to the male's container 3-4 hours after 'lightsoff', and removed after 'lights-on'. When adding or removing females, we tried to minimize disturbance to the male. However, removal of females did require some disturbance while searching through the peat although we had marked females with white paint on the pronotum to facilitate their discrimination from the male. During the sevenday treatment period, three novel females were added to a male's container every day.

Control males were maintained individually in deli containers for seven days, without access to either females or carcasses. To control for any effect that disturbances 
may have had on the other treatment groups, the control male's substrate was stirred with forceps daily at 'lights-on'. Males in the control and multiple-female treatments were fed two decapitated mealworms every three days. As males in the two carcass treatments had the opportunity to feed on carcasses, they were not fed any mealworms during the treatment period.

Following the seven days of treatments, males were transferred back into clean observation chambers with about $2 \mathrm{~cm}$ of fresh moist peat, fed half a mealworm, and placed in the darkroom to acclimate for 15-18 hours before observations began. Each male was randomly assigned a new identification number by a person other than the observer to prevent any potential observer bias during observations (i.e., post-treatment observations were made blind to treatment). Male behaviour was observed on four consecutive days post-treatment, in the same manner and under the same conditions as during the pre-treatment period.

Following the four days of observations, males were placed individually in labelled film canisters and frozen. Using a Nikon SMZ800 zoom stereoscope affixed with an Infinity 1-2 camera and NI-150 high intensity illuminator, the length and width of each male's pronotum was measured to the nearest $.01 \mu \mathrm{m}$ in NIS Elements D 3.10. Measurements were taken by capturing a still photograph of the pronotum. The pronotum width was measured at the widest part of the pronotum. Pronotum length was measured along the suture line. Each measurement was taken three times for each male, using a different still photograph for each set of measurements. 


\section{Statistical Analyses}

SAS 9.3 was used for all statistical analyses. To calculate baseline rates of signalling and searching, observations were totalled over the four pre-treatment days. Signalling and searching observations were also totalled over the post-treatment days for each male. We employed a repeated-measures, mixed-model ANCOVA, with time (preand post-treatment) as the repeated factor, family as a random effect, treatment as the main effect, and size as a covariate. We used pronotum length as our only size measurement, as we found pronotum length, pronotum width, and mass to be highly correlated. The effect of family was determined by comparing the $-2 \log$ likelihood values between models that included or excluded family as a random effect.

Repeatabilities for the signalling and searching behaviours were calculated using mean squares from general linear models as in Lessels and Boag (1987), and standard errors were calculated using the protocol from Becker (1992). The pre-treatment and post-treatment repeatability of each behaviour was calculated across all four observation days within each time period.

Results

Male beetles altered their investment in alternative mate-finding tactics after treatment. We found a significant time-by-treatment interaction for the amount of time spent signalling pre- and post-treatment (Table 1; Fig. 1). Males in the multiple-female group significantly increased time invested in signalling $\left(F_{1,174}=7.7, P=0.0061\right)$, as did males in the control group $\left(F_{1,175}=12.83, P=.0004 ;\right.$ Fig. 1$)$. Thus, males that did not 
encounter carcasses during the treatment period significantly increased investment in signalling behaviour thereafter. In the two groups that were given carcasses, however, investment in signalling was not altered (carcass: $F_{1,175}=1.48, P=0.2256$ and female and carcass: $F_{1,174}=.3, P=0.5869 ;$ Fig. 1$)$.

There was a significant difference across time periods in time spent searching, with males spending less time searching post-treatment; the time-by-treatment interaction for time spent searching was not quite significant (Table 1; Fig. 1). Pairwise comparisons revealed that males in the control, multiple-female, and carcass groups significantly decreased the amount of time spent searching after the treatment period $\left(F_{1,176}=9.57\right.$, $P=0.0023, F_{1,174}=6.67, P=0.0106$, and $\left.F_{1,176}=5.41, P=0.0212\right)$. Males in the female-andcarcass group, the only ones that were given access to both resources required for successful reproduction, did not alter their investment in searching $\left(F_{1,174}=.01\right.$, $P=0.9374$; Fig. 1).

Fig.1 illustrates how males adjusted their investment in both alternative tactics simultaneously after treatment. Males in the multiple-female treatment group both increased signalling and decreased searching following treatment, as did males in the control group. The males that were provided access to carcasses but not females, decreased their investment in searching, but did not alter their time spent signalling posttreatment. Males that were provided with females and carcasses, simulating two subsequent successful reproductive attempts, did not alter their investment in either behaviour post-treatment. 
We included family in our model as a random effect, as previous studies have shown genetic variation in the time invested in alternative mate-finding tactics (Eggert, 1992). We found a significant effect of family on both signalling $\left(X^{2}=28.1, P<0.0001\right)$ and searching $\left(X^{2}=8.3, P=0.004\right)$. Individual signalling and searching behaviour were repeatable across all trials, both pre- and post-treatment (Table 2).

Pronotum length, our measure of male body size, had a significant effect on both signalling and searching (Table 1). For signalling, there was an inverse relationship with male size, such that smaller males invested more time signalling (parameter estimate = -

$0.02622 \pm 0.0034)$. The opposite relationship was found between male size and investment in searching, as larger males spent more time searching (parameter estimate $=$ $0.01161 \pm 0.002517)$

\section{Discussion}

The results of our experiment demonstrate that male burying beetles adjust their use of alternative mate-finding tactics based on their previous experience with reproductive resources. Experimental males that did not encounter any carcasses during the treatment period significantly increased their investment in signalling while significantly decreasing investment in searching. Males in the groups that were given access to carcasses during the treatment period did not alter their investment in signalling, but males that only encountered carcasses in the absence of females decreased their investment in searching following the treatment period. Because the pre- and posttreatment observations were done under identical conditions and the beetles were not 
observed during the treatment period, these behavioural adjustments should be considered a response to prior experience rather than to the immediate conditions.

The alternative mate-finding tactics of male burying beetles are part of a conditional behavioural strategy. A male's own body size is one condition that affects his use of the alternative tactics, but environmental indicators of the profitability of each tactic are also expected to factor into the use of alternative tactics. These types of phenotypically plastic strategies are expected to evolve when the reproductive landscape is perceived as "fine-grained" (Levins, 1968), but males are able to predict the availability of mating opportunities based on some environmental cue (Shuster \& Wade, 2003). We hypothesized that the spatiotemporal distribution of reproductive resources (i.e. females and carcasses) would serve as such a cue, indicating which tactic would yield higher payoff. We found support for this hypothesis, as mate-finding tactic expression was influenced by experience with both females and carcasses. We note, however, that all males maintained both tactics to some degree post-treatment, although the relative investment in each was influenced by experience. It is likely that using a combination of both tactics is generally beneficial because rapid changes in the availability of carcasses may not be predictable based on prior experience.

Males that were allowed to mate with multiple females during the trial period subsequently increased their investment in signalling and decreased time spent searching. While we had predicted such an adjustment, we found that the control group, which did not have any interactions with females or carcasses, similarly increased signalling time 
and decreased searching time. This result suggests that a high perceived availability of females is not likely to be the cue stimulating an increased investment in the signalling tactic. It is more likely that increased investment in signalling and decreased investment in searching is triggered by a low perceived availability of carcasses. When carcasses are exceedingly rare, the payoff for searching should be low, and signalling may yield higher returns, regardless of the availability of females in the current environment.

Although the availability of females does not appear to play a role in tactic expression in the absence of carcasses, female presence does influence tactic expression when carcasses are available. While neither of the treatment groups that received carcasses altered their investment in signalling, those beetles that received only carcasses but no females decreased their searching time. Males that received both carcasses and females, however, did not show any change in searching behaviour. This suggests that the presence of females and carcasses is required for males to maintain their investment in the search tactic. The reduced time spent searching in the group that received only carcasses could be due simply to a reduction in overall activity, as well-fed burying beetles have been shown to be less active (Cordes, Kaschuba, \& Müller, 1988). However, if nutritional status did play a role, males that were on carcasses with females during the treatment period should have similarly reduced their search activity, but they did not.

We had predicted that males given both females and carcasses would subsequently increase their time spent searching and decrease signalling, as they would experience the reproductive situation of successful searchers. These males should 
perceive high availability of both reproductive resources, and thus we expected the benefits of searching to be greater than those of signalling. However, males that were allowed to mate with females on carcasses did not alter their investment in either tactic, instead continuing to search and signal at the same frequencies as they did before encountering the resources. We attribute this apparent lack of response to the fact that these males experienced the ideal reproductive situation: a carcass, a female, and no competitors. These males achieved ideal reproductive success at the tactic frequencies employed prior to the treatments, and therefore would be under no pressure to alter their strategy.

In contrast to the results of the present study, Walling et al. (2009) found no effect of experience on signalling behaviour in $N$. vespilloides. While the differences between the two studies could conceivably reflect interspecific differences in the capacity for behavioural plasticity between $N$. orbicollis and $N$. vespilloides, we think they are more likely due to differences in experimental design. In order to simulate environmental differences that might affect the profitability of the alternative tactics, we varied the males' exposure to carcasses and females for a week. In Walling et al.'s (2009) study, in contrast, the treatment that simulated different experiences for the males consisted of brief interactions with either a conspecific male or a female in the absence of any carcasses. For the mating experience treatment, Walling et al. (2009) allowed males a single mating before measuring their post-experience signalling rate. We suggest that this single mating experience is likely insufficient to alter a male's perceptions of the spatiotemporal distribution of females. We chose the design of our study based on the 
assumption that providing males with multiple females over several days would more accurately mimic the experience of successful use of the signalling tactic in the field. The lack of a significant effect of previous experience in Walling et al.'s (2009) study could also be due to the manner in which the use of tactics was measured: we observed males for six-hour periods on four days pre-treatment and another four days post-experience, whereas Walling et al.'s (2009) pre-and post-experience observations were made during a single hour on each of two consecutive days. We believe our methods may be more reflective of the natural situation, insofar as we observed males throughout their entire daily activity period and not just a small portion of it. Additionally, we were able to confirm the repeatability in male behaviour before and after treatments, ensuring that any changes were not simply due to stochastic variation.

Consistent with earlier studies in $N$. orbicollis and $N$. vespilloides (Beeler et al., 1999; Walling et al., 2009), we found an effect of absolute male size on signalling frequency, with small males signalling more than large males. We also report the novel finding of a significant positive relationship between absolute male size and time spent searching. While these size effects may be attributable to differences in competitive ability on a carcass as suggested by Beeler et al. (1999), we propose that they might also be affected by a discrepancy in the energetic costs of the two tactics. If the energetic demands of searching are greater than those of signalling, larger males with greater fat reserves should be able to invest more into searching at a lower cost than small males. While no specific data are available for our study species, flight is generally considered to be a very energetically expensive activity for insects (Reinhold, 1999; Kammer \& 
Heinrich, 1978), while pheromone production is considered relatively inexpensive. Thus, energy reserves may play an important role in a male's decision about the use of alternative tactics.

Although our study demonstrates that mating experience and the availability of reproductive resources serve as environmental cues influencing the tactic switchpoint of a conditional strategy, we do not yet know the amount of genetic variation for the switchpoint, if any. By varying the number of reproductive resources available to males, or the frequency with which they are encountered, it may be possible to determine whether males differ in the exact value of the environmental cue that is required to alter investment in alternative tactics, as predicted by the environmental threshold model (Tomkins \& Hazel, 2007). Additionally, it might be useful to investigate the effects that other social experiences, such as the presence of a competitor and non-mating interactions, have on relative investment in alternative mate-finding tactics. While Walling et al. (2009) clearly intended to simulate male interactions with conspecific competitors, it is unlikely that the brief encounters during 30 minutes of confinement with another male were sufficient to trigger adjustments in tactic use. Moreover, it is unclear what exactly the conditions were that caused males to fight each other. In our experience, male burying beetles do not engage in aggressive interactions unless a carcass or mating partner is present, and the description of the simulated "agonistic experience" in Walling et al. (2009) makes no mention that either reproductive resource was present with the two males. 
Because the alternative mate-finding tactics of male burying beetles are dependent on environmental conditions rather than being strictly genetically determined, they constitute a conditional strategy. Alternative strategies are considered to be conditional whenever the expression of tactics is contingent on non-genetic factors, but there is considerable variation among taxa in the manner in which such factors impact tactic expression. Tactic use is frequently correlated with phenotype. This can occur when a certain phenotype is fixed after a certain stage of development and constrains the subsequent use of tactics for the individual. Well-known examples include horned beetles and salmon, in which an individual's phenotype (and therefore their tactic) is determined by some threshold cue during development (Emlen, 1994; Emlen, 1997; Aubin-Horth \& Dodson, 2004). Correlations with phenotype can be looser in other systems where tactic use remains flexible, such as in horseshoe crabs in which the tactic a male employs is determined by his absolute body condition (Brockmann, 2002). In burying beetles, a male's body size clearly affects his relative use of alternative tactics. Our study confirmed earlier findings in $N$. orbicollis (Beeler et al. 1999) and $N$. vespilloides (Walling et al. 2009) that larger males tend to spend less time signalling than do smaller males, and it also showed that larger males spend more time searching than do smaller ones. As males in our study were kept singly, these findings could not have been affected by social interactions as suggested by Walling et al. (2009)

When conditional strategies are not strictly correlated with phenotype, individuals may use different tactics under different environmental or social conditions. This is commonly seen when the tactic employed is dependent on the individual's status relative 
to competitors. When larger competitors are present, males may switch to a less profitable tactic, such as satellite behaviour or female mimicry (in rove beetles: Forsyth \& Alcock, 1989; in cuttlefish: Norman, Finn, \& Tregenza, 1999; in shrimp: Correa, Baeze, Hinojosa, \& Thiel, 2003). In the grasshopper Ligurotettix coquilletti, a male's signalling behaviour is dependent on the density and proximity of conspecific males; a male is more likely to signal when alone in a bush than when sharing a bush with conspecific males (Shelley \& Greenfield, 1985). In these types of strategies, individuals are making decisions based on the current conditions and immediate social context.

In burying beetles, such behavioural adjustments based on the outcome of fights around carcasses are well documented. One recent example is a study by Carter, Head, Moore, \& Royle (2015) that investigated the signalling behaviour of competing $N$. vespilloides males on carcasses on which no female was present. These males emit pheromone in an apparent attempt to increase the attractiveness of the carcass to conspecific females, whose presence they require for successful reproduction. The males in Carter et al.'s (2015) study adjusted the location of their signalling behaviour based on their interactions with such competitors, with larger males being more likely to signal directly on the carcass. Although referred to in the study as alternative tactics, these different choices of signalling location are not equivalent to the alternative mate-finding tactics in our study, and are directly and immediately affected by the presence of competing conspecifics. In addition to differences in signalling location, males and females that lose fights over carcasses exhibit a whole host of alternative behaviours around carcasses that have been described as "satellite" behaviour for males and 
“intraspecific brood parasitism” for females (Bartlett, 1988; Eggert and Müller, 1997;

Müller et al., 1990). Subordinate individuals largely remain inactive in the soil near the carcass while exploiting opportunities to mate and oviposit around the carcass; their behaviour differs clearly from that of the winners of such interactions, and they do not stay to provide parental care (Eggert \& Müller, 1997). Even winners behave differently in the presence of competitors than when they are uncontested: female $N$. vespilloides postpone oviposition on a carcass for close to a day when a smaller female is present, which enables them to selectively destroy their competitor's offspring (Eggert \& Müller, 2011).

While behavioural adjustments in response to immediate conditions are common, adjustments can also occur based on earlier experience, as seen in our study. Prior mating experience can influence mate choice decisions in spiders (Wilder \& Rypstra, 2008; Molina \& Christenson, 2008) and lepidopterans (Fowler-Finn \& Rodriguez, 2001). In terms of conditional strategies, however, the only other example we know of where alternative mating tactics are influenced by experience is in the field cricket Teleogryllus oceanicus, in which the acoustic environment experienced by juvenile males has been shown to have a significant effect on subsequent choice of alternative mating tactics as adults (Bailey, Gray, \& Zuk, 2010). To the best of our knowledge, our study is the first to provide evidence of males adjusting their investment in alternative tactics based on experience with reproductive resources as adults. 
Aside from investment in alternative mating tactics, burying beetles also use past experiences to adjust their behaviour in other contexts. Behaviour in agonistic encounters can be affected by the outcome of earlier interactions (Otronen, 1990; Lee, Head, Carter, \& Royle, 2014). Based on the frequency of encounters with other conspecifics, $N$. orbicollis appears to assess the competitive environment, adaptively adjusting the extent of brood reduction behaviours to best match the current population density and intensity of competition (Creighton, 2005). In conjunction with our own findings, the studies mentioned above document that burying beetles possess an impressive capacity for adaptive adjustments of behaviour based on their immediate and past social context and local environment.

Funding

This work was supported by a National Science Foundation grant (IOS-1118160) to Scott K. Sakaluk and an R.D. Weigel grant from the Beta Lambda Chapter of the Phi Sigma Biological Sciences Honor Society Tess E. Piening Mulrey. 


\section{REFERENCES}

Aubin-Horth, N. \& Dodson, J.J. (2004). Influence of individual body size and variable thresholds on the incidence of a sneaker male reproductive tactic in Atlantic salmon. Evolution, 58, 136-144.

Bailey, N.W., Gray, B., \& Zuk, M. (2010). Acoustic experience shapes alternative mating tactics and reproductive investment in male field crickets. Current Biology, 20, 845-849.

Bartlett, J. (1988). Male mating success and paternal care in Nicrophorus vespilloides (Coleoptera: Silphidae). Behavioral Ecology \& Sociobiology, 23, 297-303.

Becker, W. A. (1992). Manual of Quantitative Genetics. Academic Enterprises, Pullman.

Beeler, A.E., Rauter, C.M. \& Moore, A.J. (1999). Pheromonally mediated mate attraction by males of the burying beetle Nicrophorus orbicollis: alternative calling tactics conditional on both intrinsic and extrinsic factors. Behavioral Ecology, 10, 578584.

Brockmann, H.J. (2001). The evolution of alternative strategies and tactics. Advances in the Study of Behaviour, 30, 1-51.

Brockmann, H.J. (2002). An experimental approach to alternating mating tactics in male horseshoe crabs (Limulus Polyphemus). Behavioral Ecology, 13, 232-238

Carter, M.J., Head, M.L., Moore, A.J., \& Royle, N.J. (2015). Behavioral plasticity and GxE of reproductive tactics in Nicrophorus vespilloides burying beetles. Evolution, 69, 969-978.

Cordes, R., Kaschuba, A., \& Müller, J.K. (1988). Comparison of strategies of diurnal activity of Necrophorus vespilloides (Sil., Col.) and Pterostichus oblongopunctatus (Car., Col.). Verhandlungen der Deutschen Zoologischen Gesellschaft, 81, 339-340.

Correa, C., Baeze, J.A., Hinojosa, I.A., \& Thiel, M. (2003). Male dominance hierarchy and mating tactics in the rock shrimp Rhynchocinetes typus (Decapoda: Caridae). Journal of Crustacean Biology, 23, 33-45.

Creighton, J.C. (2005). Population density, body size, and phenotypic plasticity of brood size in a burying beetle. Behavioral Ecology, 16, 1031-1036. 
Dominey, W.J. (1984). Alternative mating tactics and evolutionarily stable strategies. American Zoologist, 24, 385-396.

Eggert, A.-K. (1992). Alternative male mate-finding tactics in burying beetles. Behavioral Ecology, 3, 243-254.

Eggert, A.-K. \& Müller, J.K. (1989a). Mating success of pheromone-emitting Necrophorus males: do attracted females discriminate against resource owners? Behaviour, 110, 248-258.

Eggert, A.-K. \& Müller, J.K. (1989b). Pheromone-mediated attraction in burying beetles. Ecological Entomology, 14, 235-237.

Eggert, A.-K. \& Müller, J.K. (2011). Timing of oviposition enables dominant female burying beetles to destroy brood-parasitic young. Animal Behaviour, 82, 12271233.

Eggert, A.-K, \& Sakaluk, S.K. (2000). Benefits of communal breeding in burying beetles: a field experiment. Ecological Entomology, 25, 262-266.

Emlen, D.J. (1994). Environmental control of horn length dimorphism in the beetle Onthophagus acuminatus (Coleoptera: Scarbaeidae). Proceedings of the Royal Society B, 256, 131-136.

Emlen, D.J. (1997). Alternative reproductive tactics and male-dimorphism in the horned beetle Onthophagus acuminatus (Coleoptera: Scarabaeidae). Behavioral Ecology \& Sociobiology, 41, 335-341.

Forsyth, A. \& Alcock, J. (1989). Female mimicry and resource defense polygyny by males of a tropical rove beetle, Leistotrophus versicolor (Coleoptera: Staphylinidae). Behavioral Ecology \& Sociobiology, 26, 325-330.

Fowler-Finn, K.D. \& Rodriguez, R. (2011). Experience-mediated plasticity in mate preferences: mating assurance in a variable environment. Evolution, 66, 459-468.

Gross, M.T. (1996). Alternative reproductive strategies and tactics: diversity within the sexes. Trends in Ecology \& Evolution, 11, 92-98.

Haberer, W., Schmitt, T., Schreier, P. \& Müller, J.K. (2011). Intended and unintended receivers of the male pheromones of the burying beetles Nicrophorus humator and Nicrophorus vespilloides. Entomologia Experimentalis et Applicata, 140, 122-126.

Kammer, A.E., \& Heinrich, B. (1978). Insect flight metabolism. Advances in Insect Physiology, 13, 133-228. 
Lee, V.E., Head, M.L., Carter, M.J., \& Royle, N.J. (2014). Effects of age and experience on contest behaviour in the burying beetle, Nicrophorus vespilloides. Behavioral Ecology, 25, 172-179.

Lee, J.S.F. (2004). Alternative reproductive tactics and status-dependent selection. Behavioral Ecology, 16, 566-570.

Lessels, C.M. \& Boag, P.T. (1987). Unrepeatable repeatabilities: A common mistake. The Auk, 104, 116-121.

Levins, R. (1968). Evolution in Changing Environments: Some Theoretical Explanations. Princeton, NJ: Princeton University Press

Maynard Smith, K. (1982). Evolution and the Theory of Games. Cambridge, UK: Cambridge University Press.

Molina, Y. \& Christenson, T. (2008). Effects of mating experience on subsequent reproductive behaviour and sperm release in the spider Nesticodes rufipes. Animal Behaviour, 76, 1327-1334.

Müller, J.K., Braunisch, V., Hwang, W., \& Eggert, A.-K. (2007). Alternative tactics in individuals reproductive success in natural associations of the burying beetle, Nicrophorus vespilloides. Behavioral Ecology, 18, 196-203.

Müller, J.K. \& Eggert, A.-K. (1987). Effects of carrion-independent pheromone emission by male burying beetles (Silphidae: Necrophorus). Ethology, 76, 297-304.

Müller, J.K., Eggert, A.-K., \& Dressel, J. (1990). Intraspecific brood parasitism in the burying beetle, Nicrophorus vespilloides. Animal Behaviour, 40, 491-499.

Norman, M.D., Finn, J. \& Tregenza, T. (1999). Female impersonation as an alternative reproductive strategy in giant cuttlefish. Proceedings of the Royal Society B, 266, 1347-1349.

Otronen, M. (1990). The effect of prior experience on the outcome of fights in the burying beetle, Nicrophorus humator. Animal Behaviour, 40, 980-982.

Parker, G.A. (1982). Phenotype limited evolutionarily stable strategies. In Kings College Sociobiology Group (Eds.), Current Problems in Sociobiology (pp. 173-201). Cambridge, UK: Cambridge University Press.

Piché, J., Hutchings, J.A., \& Blanchard, W. (2008). Genetic variation in threshold reaction norms for alternative reproductive tactics in male Atlantic salmon, Salmo salar. Proceedings of the Royal Society B, 275, 1571-1575.

Pukowski, E. (1933). Okologische Untersuchungen an Necrophorus F. Zeitschrift für Morphologie und Oekologie der Tiere, 27, 518-586. 
Reinhold, K. (1999). Energetically costly behaviour and the evolution of resting metabolic rate in insects. Functional Ecology, 13, 217-224.

Sakaluk, S.K. \& Müller, J.K. (2008). Risk of sperm competition mediates copulation duration, but not paternity, of male burying beetles. Journal of Insect Behaviour, 21, 153-163.

Scott, M.P. \& Traniello, J.F.A. (1990). Behavioural and ecological correlates of male and female parental care and reproductive success in burying beetles (Nicrophorus spp.). Animal Behaviour, 39, 274-283.

Shelley, T.E. \& Greenfeild, M.D. (1985). Alternative mating strategies in a desert grasshopper: a transitional analysis. Animal Behaviour, 33, 1211-1222.

Shuster, S.M. (2010). Alternative Mating Strategies. In D.F. Westneat \& C.W. Fox (Eds.), Evolutionary Behavioral Ecology (pp. 434-450). New York: Oxford University Press.

Shuster, S.M. \& Sassman, C. (1997). Genetic interaction between male mating strategy and sex ratio in a marine isopod. Nature, 388, 373-377.

Shuster, S.M. \& Wade, M.J. (1991). Equal mating success among male reproductive strategies in a marine isopod. Nature, 350, 608-610.

Shuster, S.M. \&Wade, M.J. (2003). Mating Systems and Strategies. Princeton, NJ: Princeton University Press.

Sinervo, B. \& Lively, C.M. (1996). The rock-paper-scissors game and the evolution of alternative male strategies. Nature, 380, 240-243.

Tomkins, J.L. \& Hazel, W. (2007). The status of the conditional evolutionary stable strategy. Trends in Ecology \& Evolution, 22, 522-528.

Walling, C.A., Stamper, C.E., Salisbury, C.L. \& Moore, A.J. (2009). Experience does not alter alternative mating tactics in the burying beetle Nicrophorus vespilloides.

Behavioral Ecology, 20, 153-159.

Waltz, E.C. \& Wolf, L.L. (1984). By Jove!! Why do alternative mating tactics assume so many different forms? American Zoologist, 24, 333-343.

Wilder, S.M. \& Rypstra, A.L. (2008). Prior encounters with the opposite sex affect male and female mating behaviour in a wolf spider (Araneae, Lycosidae). Behavioral Ecology, 62, 1813-1820.

Wilson, D.S. \& Fudge, J. (1984). Burying beetles: intraspecific interactions and reproductive success in the field. Ecological Entomology, 9, 195-203. 
Table 1. Repeated Measures Mixed-Model ANCOVA F-tests for Effects of

Treatment,Time, Treatment*Time, and Pronotum Length of Signalling and Searching.

\begin{tabular}{lcccccc}
\hline \hline & \multicolumn{3}{c}{ Signalling } & \multicolumn{3}{c}{ Searching } \\
\hline Effect & $\boldsymbol{F}$ & $\mathbf{d f}$ & $\boldsymbol{P}$ & $\boldsymbol{F}$ & $\mathbf{d f}$ & $\boldsymbol{P}$ \\
\hline Treatment & 0.31 & 3,141 & 0.8170 & 0.43 & 3,140 & 0.7346 \\
Time & 12.37 & 1,174 & 0.0006 & 15.75 & 1,175 & 0.0001 \\
$\begin{array}{l}\text { Treatment* } \\
\text { Time }\end{array}$ & 3.32 & 3,174 & 0.0210 & 2.01 & 3,175 & 0.1146 \\
$\begin{array}{l}\text { Pronotum } \\
\text { Length }\end{array}$ & 59.50 & 1,174 & $<0.0001$ & 21.28 & 1,169 & $<0.0001$ \\
\hline \hline
\end{tabular}


Table 2. Signalling and Searching Were Repeatable, Both Before and After the Treatment Period.

\begin{tabular}{lcccc}
\hline \hline & \multicolumn{2}{c}{ Pre-treatment } & \multicolumn{2}{c}{ Post-treatment } \\
\hline & $\boldsymbol{R}$ & S.E. & $\boldsymbol{R}$ & S.E. \\
\cline { 2 - 5 } Signalling & 0.583 & 0.2346 & 0.562 & 0.241 \\
Searching & 0.455 & 0.2636 & 0.787 & 0.1465 \\
\hline \hline
\end{tabular}




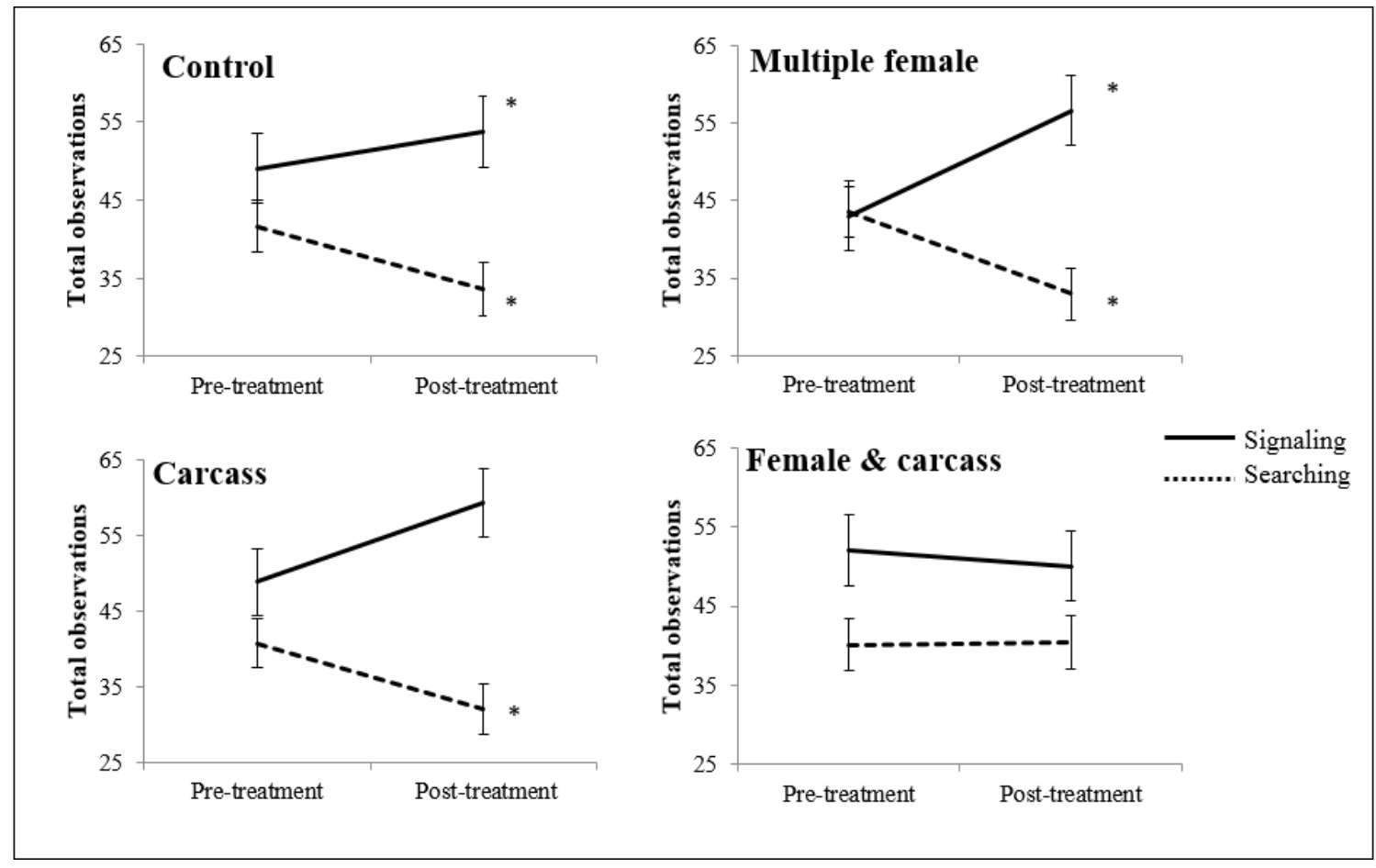

Figure 1. Total Number of Observations Spent Signaling (Solid Line) and Searching (Dashed Line) Pre- and Post-Treatment for the Four Treatment Groups. The * Indicate a Significant Difference Between Pre-Treatment and Post-Treatment Values. 